\title{
Breeding for quality improvement in turmeric (Curcuma longa L.): a review
}

\begin{abstract}
Turmeric (Curcuma longa L.) is an economically important spice and medicinal plant for production of curcuminoids, oleoresin, essential oil which are used in pharmaceutical and cosmetics industries. Presence of these contents in turmeric determine its quality. Average productivity and quality of turmeric is not satisfactory because of the poor genetic materials and non-availability of quality materials. Conventional clonal selection takes long time and slow progress to achieve the same level of quality improvement than molecular or biotechnological approaches. Use of molecular markers, transcriptome sequencing, real time PCR approaches can be applied as a supplement to conventional methods of breeding through clonal selection and advancing elite genotypes. In this paper, we will discuss in short about the use of different approaches developed for quality improvement in turmeric.
\end{abstract}

Keywords: clonal selection, curcuminoids, molecular approach, quality, turmeric
Volume 6 Issue 6 - 2017

\author{
Dipendra Kumar Ayer \\ Department of Genetics and Plant Breeding, Navsari \\ Agricultural University, India
}

\begin{abstract}
Correspondence: Dipendra Kumar Ayer, Department of Genetics and Plant Breeding, Navsari Agricultural University, Navsari, Gujarat, India, Email dip_ayer@hotmail.com
\end{abstract}

Received: January 17, 2017 | Published: April 05, 2017

\section{Introduction}

Turmeric (Curcuma longa L.) $(2 \mathrm{n}=3 \mathrm{x}=63)$ belonging to the family Zingiberaceae is an economically important spice and medicinal plant for production of curcumin, oleoresin, essential oil which are used in pharmaceutical and cosmetics industries. Traditionally turmeric is known as Haldi in India, Besar in Nepal and is under extensive cultivation in South Asian countries for medicinal, religious, culinary purposes and also as a cosmetic and dye. Dry recovery (curing percentage), curcumin and oleoresin contents determine the quality of turmeric and high variability has been observed in turmeric germplasm with respect to these characters. ${ }^{1}$ Turmeric powder obtained from rhizomes of $C$. longa is extensively used as a spice, food preservative, natural dye in food industry and in cosmetics and drugs. ${ }^{2}$ Curcuminoid, a phenylpropanoid derivative, is a mixture of curcumin $(50-60 \%$ of the curcuminoids), demethoxycurcumin and bisdemethoxycurcumin which imparts yellow colour to turmeric. ${ }^{3}$ The medicinal properties of curcuminoids as anti-inflammatory, anti-oxidant, antimutagenic, antidiabetic, anti-bacterial, hepatoprotective and expectorant are reported extensively. It is also well known in treating conditions ranging from arthritis and inflammation to Alzheimer's disease and cancer. ${ }^{2}$ Because of widespread multipurpose use of this medicinal herb in pharmacological industry, spice industry and other culinary purpose use, quality improvement for enhanced phyto-constituents production in turmeric is of great importance in the present context.

\section{Need for crop improvement in turmeric}

Although India is a leading producer of turmeric and few high yielding cultivars are available in this crop, the average productivity and quality are not satisfactory. Major problems are non-availability of requisite high yielding genotype, slow multiplication rate, low curcumin and essential oil content in available cultivars and loss due to disease during cultivation and storage. Crop improvement work in turmeric is so far confined mostly to clonal selection by exploiting the naturally occurring variations. ${ }^{1}$ To put the turmeric cultivation as an industry, it is therefore essential to develop turmeric genotypes with improved drug yielding potential containing enhanced quality and quantity of essential oil, high curcumin, high oleoresin and rhizome yield. In view of limited scope of turmeric improvement through clonal selection, role of plant biotechnology assumes significance in the present era. Several practices have already been practiced in turmeric for crop improvement viz. hybridization for recombination breeding programmes, seedling selections for advancing the clonal selections, micropropagations for disease resistant plant material generations, microrhizomes production for conservation and exchange of germplasm, in vitro pollination for disease resistant breeding. ${ }^{4,5}$ These biotechnological practices were meant to increase the biomass yield of turmeric irrespective of phytoconstituents, although some of the techniques resulted in increased curcumin content as a consequence. Thus, more promising approaches for quality improvements are still to be developed for this high value crop to meet the increasing global market demand.

\section{Genetic diversity and agroclimate based selection for elite genotypes}

The nature and magnitude of genetic divergence were estimated among sixty-five turmeric (Curcuma longa L.) genotypes using Mahalanobis D2-statistics on thirteen agro-morphological quantitative traits. ${ }^{6}$ Mahalanobis's D2 analysis revealed considerable amount of diversity among the Curcuma genotypes. Fresh and dry yield, dry recovery and curcumin content did not differ between flowered and non-flowered plants ${ }^{7}$ and thus can be assumed that flowering in turmeric would not have any impact on yield and quality. Variation in curcumin content of the same variety in different locations was also reported. ${ }^{8}$ This variation in phyto-constituent (curcumin, oleoresin and essential oils) of turmeric might be attributed to the difference in climate and soil condition of different agroclimatic zones. ${ }^{9}$ These studies suggests that breeding programs for quality improvement in turmeric need to be based on the agroclimate and diverse genetic backgrounds not on the basis of flowering and non-flowering types. Curcumin content has been found to vary depending upon soil organic carbon, available nitrogen and manganese. ${ }^{10}$ The variation in curcumin content among the genotypes under similar climatic conditions may be due to genetic factors ${ }^{11}$ indicating that phyto-constituents are 
attributes of biosynthetic gene expression pathways under the similar agroclimate. These studies suggests that multi locational niche specific breeding programs are prerequisite for quality breeding in turmeric.

Despite an ever-growing interest and commercial importance of curcumin and Curcuma essential oil, it is still not clear about nature and mode of influence by the different soil and environmental factors on the production and quality of curcumin, leaf and rhizome essential oil of this species. Variation in curcumin, leaf and rhizome oil were recorded across different agroclimatic zones. ${ }^{11}$ The volatile oil content was found to be more in C. aromatica than in C. domestica (synonym: C. longa). ${ }^{12}$ Thus, for improving quality of turmeric for curcumin and other phyto-constituents, it is necessary to maintain and conserve the genetic diversity of turmeric germplasm. It is also suggested that identification of elite genotypes of turmeric through agroclimatic zone based evaluation of important drug yielding traits is necessary ${ }^{7}$ before going for selection of parental lines for breeding programs in turmeric.

\section{Correlation and path analysis based selection for quality improvement in turmeric}

Associations of different independent and dependent traits and their cause effect relationship can be estimated by correlation and path coefficient analysis. The correlation of yield parameters with phytoconstituent content (curcumin, oleoresin, and essential oil) of selected turmeric genotypes revealed that leaf area exerted high positive significant correlation with yield and phytoconstituent content followed by tiller number. ${ }^{13}$ A positive correlation of curcumin concentrations in the seedlings and in the mature rhizomes was also found which provides a basis for the selection in the laboratory of turmeric plants high in curcumin. ${ }^{14}$ This suggests that growth and yield of turmeric have influence in phytoconstituents and while selection of parental lines, it is important to consider the association of traits for which selection is to be made. The identified promising genotypes could be useful as parental materials in cultivar and variety development with enhanced quality traits.

\section{Use of molecular markers for selection of elite turmeric genotypes}

DNA-based molecular marker techniques, viz., random amplified polymorphic DNA (RAPD) and inter simple sequence repeat (ISSR) has been most commonly used to assess the genetic diversity in turmeric genotypes. The polymorphism using both RAPD and ISSR markers demonstrated an extent of $62 \%$ correlation between the genetic similarity and geographical location. ${ }^{15}$ Development of a robust genomic microsatellite markers displayed varied levels of polymorphism. These polymorphic Simple Sequence Repeats (SSR) markers would be useful for the population genetic studies and germplasm management of turmeric. ${ }^{16}$ Knowledge on the genetic diversity of turmeric from different agro-climatic regions can be used to future breeding programs for increased curcumin, oleoresin and essential oil production to meet the ever-increasing demand of turmeric for industrial and pharmaceutical uses. ${ }^{15}$ Similarly, biochemical markers like protein, isozymes and essential oil content etc can be effectively used for in vitro detection of somaclonal variants. Over and under expression of proteins can be used to isolate variants for selection. ${ }^{17}$ Harvesting the variability among the genotypes of turmeric germ plasms for elite genotype advancement will be made easier with the help of molecular markers in breeding programs. ${ }^{18}$ used SNP analysis based on the differences in the nucleotide positions in the $177,645,724$ and a 4 base indel on the trnK gene obtained using three different lengths of $26 \mathrm{mer}, 30 \mathrm{mer}$ and $34 \mathrm{mer}$ reverse primers for the identification of four Curcuma sp. studied by $\mathrm{Xia}^{19}$ used $5 \mathrm{~S}$ rRNA spacer and chemical fingerprints for quality control and authentication of Rhizoma Curcumae, a Chinese medicine used for the removal of blood stasis and alleviating pain.

\section{Biotechnological approaches for quality improvement in turmeric}

Biotechnological approaches like micropropagation, soma clonal variation, in vitro conservation, synseed technology, protoplast fusion, production of flavor and coloring components and development of novel transgenic have great potential in conservation, utilization and increasing the production of spices. ${ }^{20}$ Efficiency of these biotechnological approaches for crop improvement is limited to propagation, conservation, safe movement and exchange of germplasm to create variability. Molecular markers and maps are being generated for crop profiling, fingerprinting, identification of duplicates, and marker assisted breeding. These marker assisted technologies along with these biotechnological propagation approaches has enhanced the improvement progress in turmeric.

Somatic embryogenesis has become one of the most desired pathways in the regeneration of plants via tissue culture because it bypasses the necessity of time-consuming and costly manipulation of individual explants, which is a problem with organogenesis. Two patterns of somatic embryogenesis are recognized: direct embryogenesis, where the embryo develops directly on the explant, and indirect embryogenesis, in which the embryo arises from a callus. Haploid technology is of significant interest for developmental and genetic research, as well as for plant breeding and biotechnology. Haploid plants are useful in understanding cellular totipotency because they develop from single male or female gametes without fertilization. ${ }^{21}$ Organogenesis and plantlet formation were achieved from the callus cultures of turmeric..$^{22,23}$ also reported plant regeneration from leaf callus of turmeric and RAPD analysis of regenerated plants showed variation at DNA level. Variants with high curcumin content were isolated from tissue cultured plantlets. ${ }^{24}$ Tissue culture techniques are important to generate the planting material free of disease pathogen and virus contamination.

\section{Molecular sequencing approaches for quality improvement in turmeric}

Gaining an understanding on the molecular mechanism underlying curcumin biosynthesis will help in improving its content and maintaining stability under all conditions of cultivation. The candidate genes for enzymes involved in curcuminoid biosynthesis has been identified from both the species viz. C. longa and $C$. aromatica. Differentially expressed genes, novel polyketide synthases, transcription factors, a large number of informative singlenucleotide polymorphisms (SNPs), simple sequence repeats (SSRs) and microRNA (miRNA) targets as resource for marker development in turmeric has been established. The rapid protocol for isolation of RNA, which works well with all the tissues of turmeric has been given by Ghansal et al., ${ }^{25}$ Ghawana et al. ${ }^{26}$ The remarkable feature of that protocol was the success in isolation of RNA with those tissues, wherein the most commonly used methods failed because of the secondary metabolites. Isolated RNA from this protocol is amenable to downstream applications such as reverse transcription-polymerase 
chain reaction (RT-PCR), differential display (DD), suppression subtractive hybridization (SSH) library construction, and northern hybridization. ${ }^{26}$

Studies in the past have indicated that curcumin content varies within accessions of $C$. long $a^{3}$ and from place to place due to the influence of environment and agro-climatic conditions ${ }^{7,9}$ which is a major concern of the spice industry. This observed difference in curcumin content is mainly due to the differential expression levels of genes encoding important enzymes of the pathway. ${ }^{27}$ Thus, an understanding of the genes involved in the biosynthesis of curcuminoids is of great significance. The transcriptome of rhizome of C. longa has already been sequenced using Illumina platform to reveal the novel transcripts related to anti-cancer and anti-malarial terpenoids. ${ }^{28}$ However, study of molecular mechanism underlying curcuminoid metabolism in turmeric, especially with respect to the full set of genes involved in this pathway and transcription factors regulating these genes, is of major significance. Mining of genes of the biosynthetic pathway has been done in several crops, and next-generation sequencing (NGS) has emerged as a cost-effective method for the detection and quantification of genes and low-abundant transcripts involved in specific biological processes. ${ }^{29} \mathrm{~A}$ study on multiple curcumin synthase pathways has identified and characterized three type III polyketide synthases, named CURS1, CURS2 and CURS3, that are capable of curcuminoid synthesis. ${ }^{30}$ These findings thus revealed the curcumin biosynthetic route in turmeric, in which diketide-CoA synthase (DCS) synthesizes feruloyldiketide-CoA, and CURS1 then converts the diketide-CoA esters into a curcuminoid scaffold, CURS2 synthesizes curcumin or demethoxycurcumin and CURS3 synthesizes curcumin, bisdemethoxycurcumin and demethoxycurcumin. The availability of the substrates and the expression levels of the three different enzymes capable of curcuminoid synthesis with different substrate specificities might influence the composition of curcuminoids in the turmeric and in different cultivars. Based on the transcriptome sequencing, type III polyketide synthase enzymes viz. curcumin synthases (CURS) and DCS have been identified and their role in curcumin synthesis has been validated through reverse transcriptase real time Polymerase Chain Reaction (RT-PCR). ${ }^{30}$ While evaluating the dynamic expression patterns, 14 unigenes were upregulated in $C$. long $a$. These upregulated genes possess potential roles in curcuminoid biosynthetic pathway namely pal, c4h, 4cl, hct, c3h, comt, dcs, curs 1 , curs 2 and curs 3 , one transcription factor (wrky), and two novel polyketide synthases (clpks 1 and clpks2) with the exception of a transcription fctaor, myb4 (Sheeja, Deepa, Santhi, \& Sasikumar, 2015). From those studies, it can be said that enhanced expression of polyketide synthase genes in $C$. longa are major contributing factors towards curcuminoids biosynthesis pathway and hence improvement in such gene expression can be achieved either by selection for such conserved expression genes family or by manipulating the gene expression pattern for such genes. ${ }^{18}$ Used sequence analysis of Chinese and Japanese Curcuma drugs on the 18S rRNA gene and trnK gene and its application based on amplification refractory mutation system analysis for their identification and authentication. . $^{1,32}$

\section{Conclusion}

Turmeric is rich in secondary metabolites or phyto-constituents such as curcuminoids, oleoresin and essential oil which are largely responsible for its pharmacological activities. Turmeric improvement through conventional clonal selection has less significance as compared to other molecular approaches for quality improvement because of the long time taking and slow progress nature of the former. Efforts have been made in biotechnology including hybridization, protoplast fusion, somaclonal variations and transgenic development to get the promising results in breeding program. Molecular approaches like marker assisted selection, molecular sequencing, gene identification, cloning and profiling have also been practiced for breeding purpose. These approaches involve less time taking and cost effective measures although requires sophisticated technology and technical skill. Thus, knowledge based on gene expression analysis, transcriptome sequencing, marker assisted selection and quantitative traits statistics can be applied in breeding programs for advancing the elite genotypes and improving phyto-constituents in turmeric genotypes. ${ }^{33}$

\section{Acknowledgements}

None.

\section{Conflict of interest}

The author declares no conflict of interest.

\section{References}

1. Khader M, Vedamuthu P, Balashanmugam P. Improvement of Turmeric In. KL Chadha, et al. editors. Advances in Horticulture, Plantation Crops and Spices. New Delhi, India: Malhotra Publishing House; 1994.

2. Krup V. Pharmacological activities of turmeric (Curcuma longa Linn): a review. Journal of Homeopathy \& Ayurvedic Medicine. 2013.

3. Thomas E, Zachariah TJ, Syamkumar S, et al. Curcuminoid profiling of Indian turmeric. J Medicinal Aromatic Pl Sci. 2011;33:36-40.

4. Peter K, Ravindran P, Nirmal Babu K, et al. Breeding of Spice Crops Black Pepper, Cardamom, Ginger and Turmeric; 2008.

5. Peter KV, Babu KN. Introduction to herbs and spices: medicinal uses and sustainable production Handbook of Herbs and Spices Second edition. Woodhead Publishing; 2012. p. 1-16.

6. Gupta AK, Mishra R, Lal RK. Genetic resources, diversity, characterization and utilization of agronomical traits in turmeric (Curcuma longa L.). Industrial Crops and Products. 2015;77:708-712.

7. Anandaraj M, Prasath D, Kandiannan K, et al. Genotype by environmen interaction effects on yield and curcumin in turmeric (Curcuma longa L.). Industrial Crops and Products. 2014;53:358-364

8. Zachariah TJ, Sasikumar B, Nirmal Babu K. Variation for quality components in ginger and turmeric and their interaction with environments. Paper presented at the Proceedings of National Seminar on Biodiversity, Conservation and Utilisation of spices, Medicinal and Aromatic Plants. Calicut: IISR; 1999.

9. Singh S, Joshi RK, Nayak S. Identification of elite genotypes of turmeric through agroclimatic zone based evaluation of important drug yielding traits. Industrial Crops and Products. 2013;43:165-171.

10. Kumar G, Reddy K, Rao M, et al. Soil and plant characters influencing curcumin content of turmeric. Indian Cocoa Arecanut and Spices Journal (India). 1992.

11. Sandeep IS, Kuanar A, Akbar A, et al. Agroclimatic zone based metabolic profiling of turmeric (Curcuma Longa L.) for phytochemical yield optimization. Industrial Crops and Products. 2016;85:229-240.

12. Ratnambal MJ. Evaluation of turmeric accessions for quality. Qualitas Plantarum Plant Foods for Human Nutrition. 1986;36(3):243-252.

13. Singh S, Sahoo S, Dash S, et al. Association of growth and yield parameters with bioactive phytoconstituents in selection of promising turmeric genotypes. Industrial Crops and Products. 2014;62:373-379. 
14. Nadgauda RS, Mascarenhas AF. A Method for Screening High Curcumin-containing Turmeric (Curcuma longa L.) Cultivars in vitro. Journal of Plant Physiology. 1986;124(3-4):359-364.

15. Singh S, Panda MK, Nayak S. Evaluation of genetic diversity in turmeric (Curcuma longa L.) using RAPD and ISSR markers. Industrial Crops and Products. 2012;37(1):284-291.

16. Siju S, Dhanya K, Syamkumar S, et al. Development, characterization and utilization of genomic microsatellite markers in turmeric (Curcuma longa L.). Biochemical Systematics and Ecology. 2010;38(4):641-646.

17. Pikulthong V, Teerakathiti T, Thamchaipenet A, et al. Development of somatic embryos for genetic transformation in Curcuma longa L. and Curcuma mangga Valeton \& Zijp. Agriculture and Natural Resources. 2016;50(4):276-285.

18. Sasaki Y, Fushimi H, Cao H, Cai SQ, Komatsu K (2002) Sequence analysis of Chinese and Japanese Curcuma drugs on the 18S rRNA gene and trnK gene and the application of amplification-refractory mutation system analysis for their authentication. Biol Pharm Bull. 25(12):15931599 .

19. Xia Q, Zhao KJ, Huang ZG, et al. Molecular genetics and chemical assessment of Rhizoma Curcumae in China. Journal of agricultural and food chemistry. 2005;53(15):6019-6026.

20. Babu KN, Divakaran M, Raj RP, et al. Biotechnological Approaches in Improvement of Spices: A Review Plant Biology and Biotechnology. 2015. p. 487-516.

21. Tapingkae T, Zulkarnain Z, Kawaguchi M, et al. 10 - Somatic (asexual) procedures (haploids, protoplasts, cell selection) and their applications A2 - Altman, Arie. In PM Hasegawa editors. Plant Biotechnology and Agriculture. San Diego: Academic Press; 2012. 141-162 p.

22. Salvi ND, George L, Eapen S. Direct regeneration of shoots from immature inflorescence cultures of turmeric. Plant cell, tissue and organ culture. 2000;62(3):235-238.

23. Salvi ND, George L, Eapen S. Plant regeneration from leaf base callus of turmeric and random amplified polymorphic DNA analysis of regenerated plants. Plant cell, tissue and organ culture. 2001;66(2):113-119.
24. Nadgauda R, Khuspe S, Mascarenhas A. Isolation of high curcumin varieties of turmeric from tissue culture. Paper presented at the Proceedings V annual symposium on plantation crops. Kasargod: CPCRI; 1982.

25. Ghansal R, Raghuvanshi S, Sharma PC. Isolation of good quality RNA from a medicinal plant seabuckthorn rich in secondary metabolites. Plant Physiol Biochem. 2009;47(11-12):1113-1115.

26. Ghawana S, Paul A, Kumar H, et al. An RNA isolation system for plant tissues rich in secondary metabolites. BMC Res Notes. 2011;4(1):85.

27. Katsuyama Y, Kita T, Funa N, et al. Curcuminoid biosynthesis by two type III polyketide synthases in the herb Curcuma longa. J Biol Chem. 2009;284(17):11160-11170.

28. Annadurai RS, Neethiraj R, Jayakumar V, et al. De Novo transcriptome assembly (NGS) of Curcuma longa L. rhizome reveals novel transcripts related to anticancer and antimalarial terpenoids. PLoS One. 2013;8(2):e56217.

29. Kalra S, Puniya BL, Kulshreshtha D, et al. De Novo transcriptome sequencing reveals important molecular networks and metabolic pathways of the plant, Chlorophytum borivilianum. PLoS One. 2013;8(12):e83336.

30. Katsuyama Y, Kita T, Horinouchi S. Identification and characterization of multiple curcumin synthases from the herb Curcuma longa. FEBS Lett. 2009;583(17):2799-2803.

31. Sasaki Y, Fushimi H, Komatsu K. Application of single-nucleotide polymorphism analysis of the trnK gene to the identification of Curcuma plants. Biol Pharm Bull. 2004;27(1):144-146.

32. Sasikumar B. Genetic resources of Curcuma:diversity, characterization and utilization. Plant Genetic Resources: characterization and utilization. 2005;3(2):230-251.

33. Sheeja TE, Deepa K, Santhi R, et al. comparative transcriptome analysis of two species of curcuma contrasting in a high-value compound curcumin: insights into genetic basis and regulation of biosynthesis. Plant Molecular Biology Reporter. 2015;33(6):1825-1836. 